\title{
CONSULTORIO PSICOLÓGICO VIRTUAL: SOPORTE PARA LA POBLACIÓN ACTUAL
}

Virtual psychological consultation: Support for the current population

Edgar Rodríguez Vílchez*

\begin{abstract}
Resumen
Con el pasar de los años se ha tomado mayor importancia a la salud mental, actualmente existen instituciones encargadas de promocionar actividades o eventos a favor del bienestar mental de las personas. Aunque también existen alternativas desde hace varios años, como es el caso de la consejería y orientación, este tipo de atenciones ha servido para enlazarse con la tecnología, convirtiéndose en consultorios psicológicos virtuales, destinados a las personas que no puedan asistir a una consultar "cara a cara". En nuestro país existe este tipo de soporte que es respaldado por Universia, y atendido por un equipo de psicólogas de la Unifé, desde hace 10 años aproximadamente, donde las personas siguen acudiendo para comunicar su motivo de consulta esperando una respuesta que los guie y oriente a encontrar algún tipo de alternativa para mejorar la dificultad que presentan. Entre los motivos de consultas más frecuentes, en estos tres años (2010, 2011 y 2012), se encuentran presentes: adicciones (ludopatía y consumo de sustancias psicoactivas), problemas familiares, trastornos en el estado de ánimo y orientación vocacional.
\end{abstract}

Palabras clave: terapia psicológica virtual

\begin{abstract}
With the passing of years, the mental health has taken greater importance, now there are institutions that promote activities or events in favor for the mental well-being of people. Although there are alternatives since several years ago, such as counseling and guidance, this type of care has served to connect with technology, becoming virtual psychological clinics, for people who can't attend a consultation "face to face". In our country there are these kinds of support that is supported by "Universia", and attended by a team of Unife's psychologists, from about ten years ago, where people continue attending to communicate their consultation motive and waiting for a response that lead and guide to find some type of alternative to improve their difficulty. In the past three years (2010, 2011 and 2012), among the most common consultation motives there are: addictions (compulsive gambling and consumption of psychoactive substances), family problems, mood disorders and vocational training.
\end{abstract}

Keywords: psychological therapy on line

* Docente de la Universidad Femenina del Sagrado Corazón. Director del Instituto Peruano de Psicoterapia Cognitiva Conductual. erodriguez@unife.edu.pe / erodriguez_vilchez@hotmail.com 


\section{INTRODUCCIÓN}

Debido a los avances tecnológicos se ha visto la necesidad de crear nuevos espacios de apoyo, por ello hace once años (2002) se introdujo el consultorio psicológico virtual dirigido por un equipo de psicólogos(as) capacitados de la Universidad Femenina del Sagrado Corazón (UNIFÉ) en convenio con Universia Perú, para brindar soporte a la población sobre todo a quienes navegan en el internet y que requieren alguna orientación por temas de interés o de preocupación.

Entre los temas de mayor concurrencia, se han encontrado consultas de personas con consumo de sustancias psicoactivas o ludopatía sobre todo de familiares solicitando orientación por sentirse impotentes ante la situación que atraviesan; también, adolescentes con necesidades de orientación vocacional que generaba en ellos intranquilidad por su futuro; además, jóvenes (sobre todo mujeres) que padecen de violencia de pareja y/o por temas de celos, entre otros.

De acuerdo a las consultas recepcionadas por los especialistas, la mayoría de las personas que deseaban resolver las dificultades presentadas, no acuden a consulta psicológica ya sea privada o en alguna entidad pública. Se podría considerar como algunos de los factores: la falta de información ante el tema de consulta, la escasa importancia brindada a una atención psicológica, la necesidad de buscar un soporte rápido y adecuado, el miedo a una respuesta indeseada o la falta de recursos económicos.

\section{MARCO TEÓRICO}

De acuerdo a la historia, en los noventas se experimentó la llegada del internet gracias a la tecnología, esta evolución ha sentado un gran cambio en la posibilidad de acceder a la información rápidamente, ello se ha conservado hasta la actualidad con un manejo no sólo por parte de los adultos, sino también de los adolescentes y niños(as).

Se podría considerar al internet como un aliado, en este caso, positivo, que abrirá la puerta de navegación en búsqueda de orientación a quienes acudan a consultar virtualmente, utilizando herramientas importantes, como: el foro, el chat y la lectura de artículos
Universia Perú (2007). En este caso entraría a tallar el consultorio psicológico virtual propiamente dicho, el cual es denominando como: "un espacio de encuentro con especialistas en diferentes disciplinas que te permitirá tener mayor información sobre los temas tratados en cada consultorio".

Guevara (2008). “...el consultorio psicológico virtual se presenta como una alternativa para aliviar tensiones y recibir apoyo que le permita encontrar un camino frente a las adversidades cotidianas".

Para Vallejo (2003), es importante la utilización del internet en conjunto con la atención psicológica que se brinda a los pacientes:

La creación de una comunidad virtual de apoyo, es muy útil. Este soporte es extensivo a trastornos como la depresión o el riesgo de suicidio. La posibilidad de contar con apoyo de otros pacientes y de profesionales mejora significativamente el seguimiento de estos pacientes.

Según la Real Academia Española (2010) define soporte como todo apoyo o sostén. ¿Qué sucede cuando este espacio de interacción virtual se convierte útil para las personas que necesitan soporte? En consecuencia y según lo observado en el consultorio psicológico virtual, se podría denominar como soporte al espacio virtual que genera bienestar para salvaguardar la salud mental de los consultantes.

Vallejo (2003). Hace referencia a un programa denominado "telesalud" que son diseñados para personas discapacitadas, pacientes que residen en zonas rurales o con enfermedades crónicas, quienes no pueden desplazarse hasta el consultorio de un especialista. "Puede considerarse que el uso de la "telesalud" es tan efectivo como la asistencia convencional cara a cara. No obstante, no se pretende que la "telesalud" sustituya al sistema de asistencia convencional sino que lo complemente...".

Debido a los precedentes sentados por consultorios psicológicos virtuales. Para Vallejo y Jordán (2007) se considera como una opción sólida la psicoterapia a pacientes que presentan fobias, trastornos de pánico y trastornos de ansiedad: 
Los múltiples estudios de orientación cognitivo conductual sobre el tratamiento de los problemas relacionados con el pánico y la ansiedad (fobias simples, fobia social, agorafobia, crisis de angustia) han dado lugar a protocolos de intervención eficaces y muy estructurados. Las intervenciones bien pautadas son las más proclives a su adaptación a un programa de ordenador y así los programas más numerosos son los aplicados a trastornos fóbicos y de pánico.

Vallejo (2003). "Resulta necesario, en consecuencia, asistir de forma activa, con iniciativas, a este nuevo mundo de posibilidades diversas, ofreciendo experiencias concretas en las que los aspectos psicológicos han de quedar integrados en planteamientos más amplios y comprehensivos de la salud."

\section{OBJETIVOS}

- Orientar gratuitamente a las personas que visitan el consultorio psicológico virtual.

- Absolver las consultas que presenten sin confundir con psicoterapia.
- Contribuir con la comunidad brindando soporte a la población en general.

\section{MODALIDAD DE ATENCIÓN}

Foro: Es un espacio virtual donde las personas escriben las dificultades que presentan, posteriormente, el especialista a cargo lee cada una de ellas y responde brindando orientación al consultante.

Artículo: Se publican artículos en la página web de diversos temas de interés, los cuales están al alcance de las personas que navegan para que puedan ser leídos y de esta manera informar de manera adecuada.

\section{VENTAJAS Y DESVENTAJAS}

Según lo observado en el consultorio psicológico virtual, se han encontrado tanto ventajas como desventajas que son importantes tenerlas en cuenta para su revisión.

\begin{tabular}{|c|c|}
\hline VENTAJAS & DESVENTAJAS \\
\hline $\begin{array}{l}\text { - Accesibilidad virtual a bajo costo } \\
\text { económico. } \\
\text { - Contar con el anonimato o un sobrenombre. } \\
\text { - } \quad \text { Facilidad para consultar sobre tema de interés } \\
\text { o preocupación } \\
\text { - Temor en acudir a un especialista y escuchar } \\
\text { una respuesta indeseable. } \\
\text { - Expresar libremente sus emociones e ideas } \\
\text { sin ser juzgados. } \\
\text { El tiempo de la consulta es corto en } \\
\text { comparación con la atención psicológica } \\
\text { individual. }\end{array}$ & $\begin{array}{l}\text { - La respuesta no es inmediata. } \\
\text { - La identificación del consultante no es sólida } \\
\text { - } \quad \text { tampoco la certificación del especialista. } \\
\text { ausente. } \\
\text { - Las emociones de los pacientes no se movilizan } \\
\text { como en terapia. } \\
\text { - Eventos claves no se mencionan por el corto } \\
\text { tiempo o por no estar cara a cara. } \\
\text { - Los datos proporcionados pueden ser falsos. }\end{array}$ \\
\hline
\end{tabular}




\section{TEMAS CONSULTADOS}

- Ludopatía

- Problemas familiares

- Orientación vocacional

- Problemas en la pareja

- Estado de ánimo

- Consumo de sustancias psicoactivas

- Problemas en el trabajo

- Trastorno alimenticio

- Trastorno de la personalidad

\section{ANÁLISIS ESTADÍSTICOS}

\section{Población:}

En la población atendida en el consultorio virtual, se puede observar que por año existe un incremento en el número de personas consultantes en el foro de Universia. En el año 2011 podemos observar que fueron atendidas más personas a comparación de las atenciones en los años 2012 y 2010, existiendo este último año un menor número de atenciones (Ver Tabla 1)

\section{Tabla 1}

Números de personas atendidas del 2010 al 2012

\begin{tabular}{cc}
\hline Año & Foro \\
\hline 2010 & 68 \\
2011 & 94 \\
2012 & 89 \\
Total & $\mathbf{2 5 1}$ \\
\hline
\end{tabular}

\section{Motivos de consultas}

Al referirnos a motivo de consulta de quienes participaron en el foro; durante el año 2011 podemos observar mayor porcentaje de personas atendidas en adicciones y estados de ánimo, así como en los problemas familiares. Un similar resultado encontramos en el año 2010, ya que, las consultas de mayor frecuencia fueron en los temas sobre adicciones y problemas familiares, así como en estado de ánimo. Aunque en el año 2012, existe un porcentaje mayor en adicciones, podemos observar que el motivo de consulta de orientación vocacional y estados de ánimo son, también, los temas más consultados a diferencia de los otros dos años. Por lo tanto, existe un gran porcentaje de personas consultantes en los temas de adicciones y estados de ánimo, ocupando el tercer lugar los problemas familiares, siguiéndole el tema de orientación vocacional. (Ver Tabla 2)

Tabla 2

Atenciones en porcentajes considerando el motivo de consulta, del año 2010 al 2012

\begin{tabular}{lccc}
\hline & $\mathbf{2 0 1 0}(\boldsymbol{\%})$ & $\mathbf{2 0 1 1}(\boldsymbol{\%})$ & $\mathbf{2 0 1 2}(\boldsymbol{\%})$ \\
\hline Adicciones & $40 \%$ & $40 \%$ & $35 \%$ \\
Problemas Familiares & $16 \%$ & $13 \%$ & $10 \%$ \\
Estado de ánimo & $13 \%$ & $19 \%$ & $16 \%$ \\
Orientación Vocacional & $6 \%$ & $10 \%$ & $19 \%$ \\
Problemas en relación conyugal & $12 \%$ & $4 \%$ & $9 \%$ \\
Problemas laborales & $9 \%$ & $7 \%$ & $6 \%$ \\
Trastorno alimenticio & $3 \%$ & $4 \%$ & $3 \%$ \\
Trastorno de la personalidad & $1 \%$ & $2 \%$ & $2 \%$ \\
Total & $100 \%$ & $100 \%$ & $100 \%$ \\
\hline
\end{tabular}

\section{REFERENCIAS}

Guevara, M. (2008). Consultorio Psicológico Virtual: Una alternativa preventiva para la psicología. Revista Avances en Psicología. 16 (1). Unifé, Lima 53-64

Real Academia Española. (2010). Diccionario de la Lengua Española. Recuperado de http://lema.rae. es/drae/?val=soporte

Universia Perú. (2007). Consultorio Psicológico Virtual. Perú. Recuperado de http://consultorios. universia.edu.pe/que-es-un-consultorio-virtual/

Vallejo, M. (2003). Nuevas Tecnologías, Internet y Psicología Clínica. Infocop, 86. Recuperado de http://www.cop.es/infocop/vernumero. asp?id=1116

Vallejo, M. y Jordán C. (2007). Psicoterapia a través de internet. Recursos tecnológicos en la práctica de la psicoterapia. Boletín de Psicología 91 (11). 27-42. Recuperado de http://www.uv.es/seoane/ boletin/previos/N91-2.pdf

Fecha de recepción: 17 de junio 2013

Fecha de aceptación: 30 de Septiembre 2013 